\title{
A Broadband and High-Gain Metamaterial Microstrip Antenna
}

\author{
Le-Wei Li, ${ }^{1,}$ a) Ya-Nan Li, ${ }^{2}$ Tat Soon Yeo, ${ }^{2}$ Juan R. Mosig, ${ }^{3}$ and Olivier J.F. Martin ${ }^{3}$ \\ ${ }^{1)}$ Institute of Electromagnetics, University of Electronic Science and Technology of China, Chengdu, \\ China 611731. \\ ${ }^{2)}$ Department of Electrical and Computer Engineering, National University of Singapore, Kent Ridge, \\ Singapore \\ ${ }^{3)}$ Institut de Transmissions, Ondes et Photonique (ITOP), Swiss Federal Institute of Technology, Lausanne, \\ Switzerland
}

(Dated: 30 March 2010)

A braod bandwidth and high gain rectangular patch antenna was specifically designed in this paper using planar-patterned metamaterial concepts. Based on an ordinary patch antenna, the antenna has isolated triangle gaps and crossed strip-line gaps etched on the metal patch and ground plane, respectively. Demonstrated to have left-handed characteristics, the patterned metal patch and finite ground plane form a coupled capacitive-inductive circuit of negative index metamaterial. It is shown to have great impact on the antenna performance enhancement in terms of the bandwidth significantly broadened from a few hundred $\mathrm{MHz}$ to a few $\mathrm{GHz}$, and also in terms of high efficiency, low loss and low voltage standing wave ratio. Experimental data show a reasonably good agreement between the simulation and measured results. This antenna has strong radiation in the horizontal direction for some specifical applications within the entire band.

PACS numbers: 84.40.Ba, 28.52.Fa, 84.40.-x, 84.40.Lj

A microstrip patch antenna ${ }^{1,2}$ represents one of the most commonly utilized printed antennas in practice. It enjoys its advantages of low profile, simple structure, low cost, and omnidirectional radiation patterns ${ }^{1,2}$. A narrow bandwidth is, however, the main drawback of the microstrip patch antennas. Some approaches have been therefore developed for bandwidth enhancement ${ }^{3-6}$. Among those common ones, one is to increase the height of the dielectric substrate while the other is to decrease the substrate dielectric constant. Certainly, the latter will induce the matching circuits to be impractical due to excessively wide lines designed.

Since the artificial left-handed materials (LHMs) or metamaterials were proposed, theoretically characterized, and experimentally realized ${ }^{7-13}$, scientists and engineers have tried various ways to bring these special material characteristics into practical applications. The metamaterials have been successfully applied in optical frequency band for optical imaging ${ }^{14-17}$. Although it is easier to realize metamaterials in microwave frequency region for negative refractions, there was still little progress toward practical applications ${ }^{18}$. At microwave frequencies, potential applications include primarily (a) substrate materials for antenna and microwave component designs and fabrications, and (b) absorbing materials for engineering and radar applications. For example, split ring resonators $(\mathrm{SRRs})^{11,19-21}$ and some other planar structures ${ }^{22-24}$ were applied in some antenna fabrications to minimize the size and enhance the radiation. Also in some other designs, artificial magnetic materials ${ }^{10,25}$ with

\footnotetext{
a) Also with Department of Electrical \& Computer Engineering, National University of Singapore, Kent Ridge, Singapore; Electronic mail: lwli@nus.edu.sg http://www.ece.nus.edu.sg/lwli
}

stacks of SRRs under patch antenna were proposed and it was found that the resonant frequency of the original patch antenna can be significantly reduced ${ }^{26}$. There are, however, still primarily fundamental issues at microwave frequencies: narrow bandwidth (when both negative permittivity and negative permeability merge in the same band) and high loss (due to the ohmic loss and radiation loss of inclusion elements), and this drawback becomes especially serious when the SRR- and other inclusiontypes of metamaterials are used as substrate of the patch antenna.

The objective of this paper is thus to enhance, in a completely different approach, the bandwidth and gain of a conventional patch antenna by applying the planar metamaterial patterned structures directly on the upper patch and bottom ground of the dielectric substrate, so the patch antenna can have an excellent performance.

A conventional microstrip patch antenna is usually mounted on a substrate and backed by a conducting ground plane. In the present investigation, as shown in Fig. 1, a planar left-handed material pattern on the rectangular patch antenna mounted on the substrate is designed to enhance its horizontal radiation as well as to broaden its working bandwidth via its coupling with the conducting ground backed to the substrate and patterned in a different way. On the upper patch, the periodic gaps are designed in the form of isolated micro-triangles; while on the bottom ground plane, as shown in Fig. 1(a), periodically distributed cross strip-line gaps are designed. To maintain the transmission consistency of input energy, the metal in and around the feed-line area is, however, not etched. The prototype and the dimension of the two patterned planes of the proposed antenna are shown in Fig. 1. The left-handed characteristics of these patterns were already demonstrated $\mathrm{in}^{27}$ and thus will not be further discussed here. We have also optimized the original 
patterns slightly to achieve a better performance of the antenna. Physically, the upper patch and bottom plane are coupled to form a capacitive-inductive (C-L) equivalent circuit and thus can induce backward wave which travels along the plane of patch. In this connection, the radiation along the patch direction is significantly enhanced.

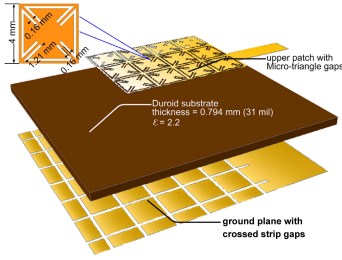

(a) 3D view

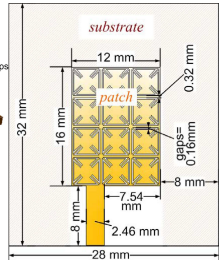

(b) Top view

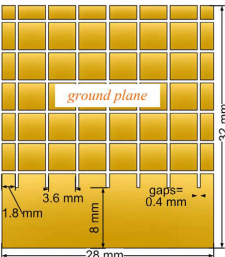

(c) Bottom view
FIG. 1. (Color online) Configuration in 3D of the proposed patch antenna.

A conventional antenna of the same size is used as a reference for comparison and both the conventional rectangular patch and the proposed patch antenna are fed by an off-centered microstrip line. The substrate used here is Duroid with a relative permittivity of $\varepsilon_{r}=2.2$, and its thickness is 31 mil. The area of the upper patch mounted is $12 \times 16 \mathrm{~mm}^{2}$. Two different widths $g$ of the gaps (i.e., $0.3 \mathrm{~mm}$ and $0.4 \mathrm{~mm}$ ) on the ground plane are assumed. Therefore, different inductance components of the LHM characteristics can be obtained so as to control the resonance frequency and also the working bandwidth of the designed antenna.

The prototypes LHM patch antenna was numerically simulated/designed, physically fabricated, practically measured and comparatively studied with theoretical results. To optimize it for various parameters, a full wave finite element method simulator was used. The computed $S_{11}$ values of the proposed antenna and the reference patch antenna are obtained and shown in Fig. 2(a). As seen, the working bandwidth of the conventional patch antenna is $200 \mathrm{MHz}$ (between $7.1 \mathrm{GHz}$ and $7.3 \mathrm{GHz}$ ), which is typically very narrow as expected but serves as a benchmark for the improved designs. The proposed antenna is designed to have the $0.4 \mathrm{~mm}$ gap at the bottom, and the $-10 \mathrm{~dB}$ bandwidth (which is standardly defined for engineering applications) falls within $5.3 \mathrm{GHz}$ and $8.5 \mathrm{GHz}$ (which is $3.2 \mathrm{GHz}$ in bandwidth, and is 16 times wider than the conventional antenna). When the gap at the bottom becomes $0.3 \mathrm{~mm}$, the -10 $\mathrm{dB}$ bandwidth turns within $5.7 \mathrm{GHz}$ and $8.6 \mathrm{GHz}$ (which is $2.9 \mathrm{GHz}$ in bandwidth, and is 14.5 times wider than the conventional antenna).

To verify the accuracy of designs, two proposed antennas with different gap widths (one of which is shown in Fig. 3) are then fabricated and measured. Experimental results of $S_{11}$ values are compared with numerically predicted results in Fig. 2(b). The general variational trace of the experimental results follows closely to that of the simulated $S_{11}$ values. From the -10 -dB level line, it is seen that a reasonably good agreement between the de-
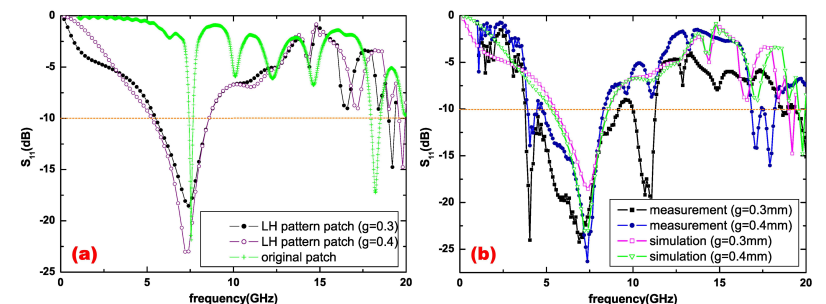

FIG. 2. (Color online) S-parameter values of the proposed antenna with different gap widths. (a) Computed $S_{11}$ values for metamaterial antenna and conventioal patch. (b) Measured and computed $S_{11}$ values for metamaterial antennas.

sign working bandwidth and the measured bandwidth is found. In fact, the fabricated antennas have even wider bandwidths than those of modeled antennas.

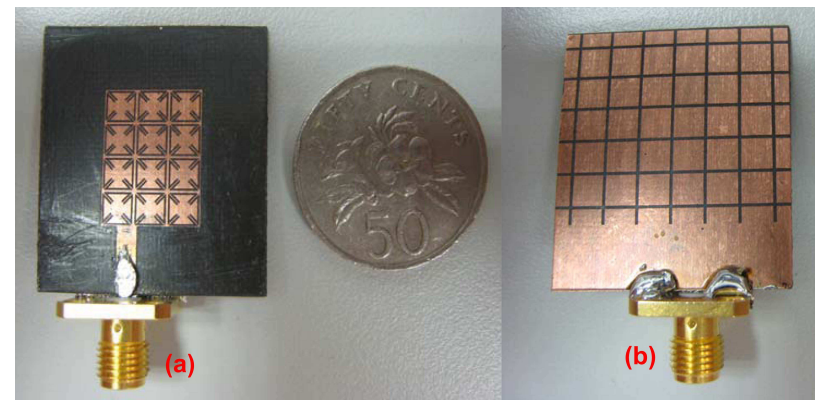

FIG. 3. (Color online) (a) Top and (b) bottom views of a fabricated patch antenna.

Antenna gains are measured within the entire frequency band as shown in Fig. 4(a). The antenna gain is generally above $4 \mathrm{~dB}$ with the peak of $7.2 \mathrm{~dB}$. For a patch antenna, this has been a very high as compared with that of a standard one. The voltage standing wave
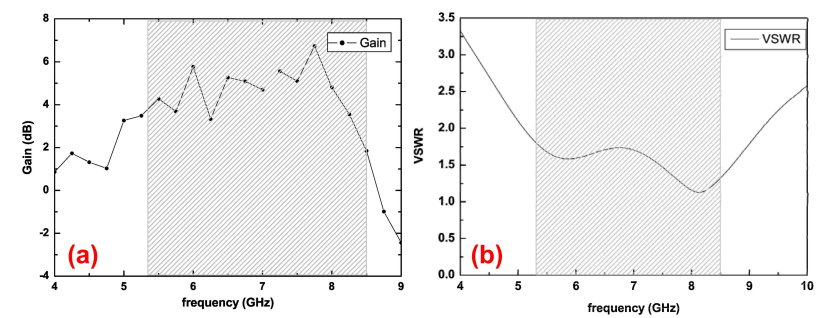

FIG. 4. (Color online) (a) Measured gain and (b) simulated VSWR of proposed antenna with a gap of $0.4 \mathrm{~mm}$. Shaded area shows the working band.

ratio (VSWR) is defined as $(1+|\Gamma|) /(1-|\Gamma|)$ where $\Gamma$ denotes the reflection coefficient. The VSWR value serves as a good measure to check if the system is working efficiently. In the present work, the simulated VSWR value is well below 2 (which serves as a good reference value for most of the engineering applications) within the frequency band as shown in Fig. 4(b).

Due to the left-handed transmission characteristics, the wave propagation along the patch induces the strongest radiation in horizontal direction instead of the vertical direction of the conventional patch antenna. To 
further confirm this, two frequencies, i.e., $6.66 \mathrm{GHz}$ and $7.77 \mathrm{GHz}$ both in the working bandwidth, are randomly chosen to characterize radiation of the antenna. According to the computed results, the $3 \mathrm{D}$ radiation patterns at these two frequencies are shown in Fig. 5. It is apparent that energy radiates to the horizontal directions.

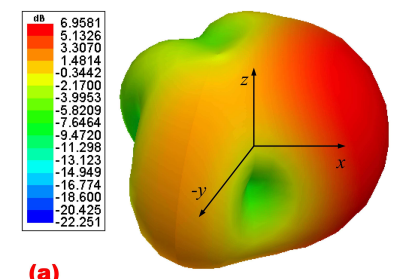

(a)

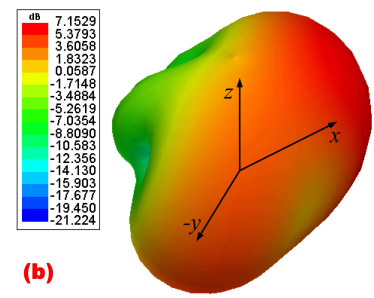

FIG. 5. (Color online) Computed 3-D radiation patterns at (a) $6.66 \mathrm{GHz}$ and (b) $7.77 \mathrm{GHz}$, respectively.

To further verify the results, the measured copolarization and cross-polarization radiation patterns are plotted in 2D in Fig. 6, respectively. The gain is found to be able to reach as high as $7.14 \mathrm{~dB}$, which is quite desirable for a single patch and it would never happen if a conventional patch antenna is designed. Also seen from the $2 \mathrm{D}$ patterns at both of these randomly picked frequencies, the radiated energy is mainly focused in the $x$-direction in the case of the co-polarization. In the case of the cross-polarization, the radiation level is well suppressed except at around $210^{\circ}$ of the $\theta$-direction in the $x y$-plane. So we may also take a good advantage of this special characteristic to transmit two quadrature signals using the antenna as a directional one for beam control.

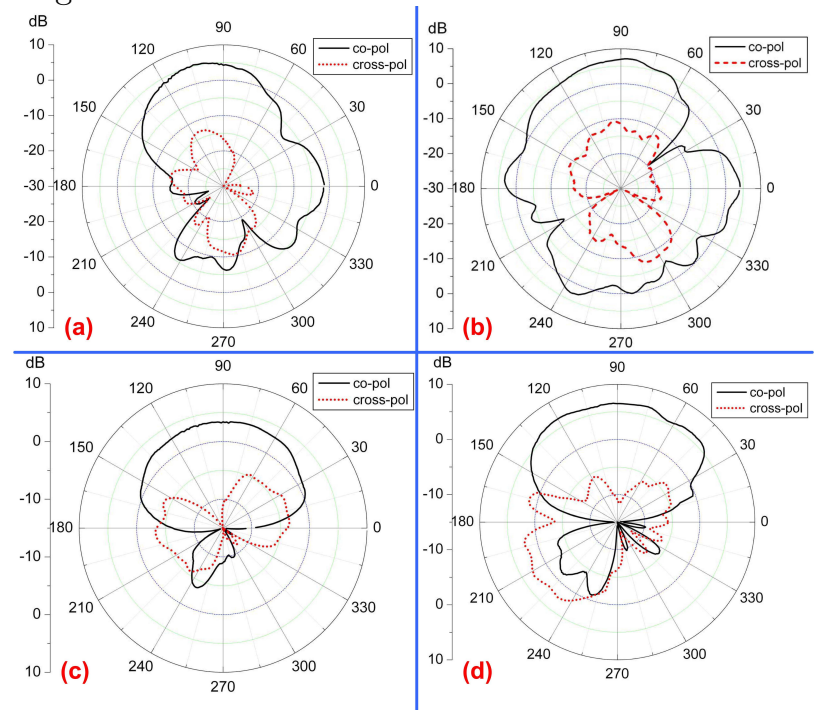

FIG. 6. (Color online) Measured radiation patterns of copolarization (solid line) and cross-polarization (dotted line) (a) at $6.66 \mathrm{GHz}$ in the $x y$-plane; (b) at $7.77 \mathrm{GHz}$ in the $x y$ plane; (c) at $6.66 \mathrm{GHz}$ in the $y z$-plane; and (d) at $7.77 \mathrm{GHz}$ and in the $y z$-plane, respectively.

In summary, a broad-bandwidth and high-gain patch antenna is designed and fabricated using the metamaterial concept via the pattern-etched upper patch and bottom ground plane. The working frequency bandwidth of the rectangular patch antenna is significantly broadened from about $200 \mathrm{MHz}$ to about $3 \mathrm{GHz}$ (at about 15 times). Also, this patch antenna designed using the metamaterial concept has very high efficiency of above $98 \%$ according to simulation, very low loss (or high gain according to measurements) and low voltage standing wave ratio.

The authors are grateful to the Defence Science and Technology Agency of Singapore and the National University of Singapore for the financial support in terms of Defence Innovative Research Program with Project No: DSTA-NUS-DIRP/2007/02. This work has been partially supported by the AFOSR/AOARD Projects: AOARD-07-4024 and AOARD-09-4069.

${ }^{1}$ C. A. Balanis, Antenna Theory: Analysis and Design (John Wiley \& Sons., 1982).

${ }^{2}$ R. Bancroft, Microstrip and Printed Antenna Design (Noble Publishing, 2004).

${ }^{3}$ P. Li, H. W. Lai, K. M. Luk, and K. Lau, IEEE Antennas Wireless Propagat. Lett. 3, 211 (2004).

${ }^{4}$ Y. Chang and R. F. Harrington, IEEE Trans. Antennas Propagat. 25, 789 (1977).

${ }^{5}$ S. Enoch, G. Tayeb, P. Sabouroux, N. Guérin, and P. Vincent, Phys. Rev. Lett. 89, 213902 (2002).

${ }^{6}$ L. Yang, M. Fan, F. Chen, J. She, and Z. Feng, IEEE Trans. Microwave Theory Tech. 53, 183 (2005).

${ }^{7}$ V. G. Veselago, Sov. Phys. Usp. 10, 509 (1968)

${ }^{8}$ J. B. Pendry, A. J. Holden, and W. J. S. D. J. Robbins, IEEE

Trans. Microwave Theory Tech. 47, 2075 (1999).

${ }^{9}$ J. B. Pendry, Phys. Rev. Lett. 85, 3966 (2000).

${ }^{10}$ D. R. Smith, W. J. Padilla, D. C. Vier, S. C. Nemat-Nasser, and S. Schultz, Phys. Rev. Lett. 84, 4184 (2000).

${ }^{11}$ D. R. Smith and D. Schurig, Phys. Rev. Lett. 90, 077405 (2003).

${ }^{12}$ C.-W. Qiu, H.-Y. Yao, L.-W. Li, S. Zouhdi, and T.-S. Yeo, Phys. Rev. B 75, 155120 (2007).

${ }^{13}$ C.-W. Qiu, H.-Y. Yao, L.-W. Li, S. Zouhdi, and T.-S. Yeo, Phys. Rev. B 75, 245214 (2007).

${ }^{14}$ N. Fang, H. Lee, C. Sun, and X. Zhang, Science 308, 534 (2005).

${ }^{15}$ J. B. Pendry, D. Schurig and D. R. Smith, Science 312, 1780 (2006).

${ }^{16}$ S. Zhang, W. Fan, N. C. Panoiu, K. J. Malloy, R. M. Osgood, and S. R. Brueck, Phys. Rev. Lett. 95, 137404 (2005).

${ }^{17}$ F. J. García de Abajo and J. J. Sáenz, Phys. Rev. Lett. 95, 233901 (2005).

${ }^{18}$ D. Schurig, J. J. Mock, B. J. Justice, S. A. Cummer, J. B. Pendry, A. F. Starr, and D. R. Smith, Science 314, 977 (2006).

${ }^{19}$ X. Chen, B. I. Wu, J. A. Kong, and T. M. Grzegorczyk, Phys. Rev. E 71, 046610 (2005).

${ }^{20}$ H.-Y. Yao, W. Xu, L.-W. Li, Q. Wu, and T.-S. Yeo, IEEE Trans. Microwave Theory Tech. 53, 1469 (2005).

${ }^{21}$ P. Gay-Balmaz and O. J. Martin, Appl. Phys. Lett. 81, 939 (2002).

${ }^{22}$ F. Falcone, T. Lopetegi, M. A. Laso, J. D. Baena, J. Bonache, M. Beruete, R. Marques, F. Martin, and M. Sorolla, Phys. Rev. Lett. 93, 197401 (2004).

${ }^{23}$ H.-T. Chen, J. F. O'Hara, A. J. Taylor, R. D. Averitt, C. Highstrete, M. Lee, and W. J. Padilla, Opt. Express 15, 1084-1095 (2007).

${ }^{24}$ A. Sanada, C. Caloz, and T. Itoh, IEEE Trans. Microwave Theory Tech. 52, 1252 (2004).

${ }^{25}$ H.-Y. Yao, L.-W. Li, Q. Wu, and J. A. Kong, Progress In Electromagnetics Research 51, 197 (2005).

${ }^{26}$ W. Xu, L.-W. Li, H.-Y. Yao, T.-S. Yeo, , and Q. Wu, J. Electromagn. Waves Appl. 19, 2033 (2005).

${ }^{27}$ N. Matsunaga, A. Sanada, and H. Kubo, IEICE Trans. Electron. E89-C, 1276 (2006). 